\section{Clase social, factores de riesgo psicosocial en el trabajo y su asociación con la salud autopercibida y mental en Chile}

\author{
Social class, psychosocial occupational risk factors, \\ and the association with self-rated health and \\ mental health in Chile
}

\author{
Classe social, fatores psicossociais de risco \\ ocupacional e sua associação com saúde \\ autopercebida e mental no Chile
}

Kátia Bones Rocha 1

Carles Muntaner 2

Orielle Solar 3

Carme Borrell 4

Pamela Bernales 3

María José González 3

Ciro Ibañez 3

Joan Benach 5

Clélia Vallebuona 6

\author{
1 Pontifícia Universidade \\ Católica do Rio Grande do \\ Sul, Porto Alegre, Brasil. \\ 2 University of Toronto, \\ Toronto, Canada. \\ 3 Universidad Mayor \\ Santiago, Chile. \\ 4 Agéncia de Salut Pública \\ de Barcelona, Barcelona, \\ España. \\ 5 Universitat Pompeu Fabra, \\ Barcelona, España \\ 6 Ministerio de Salud de \\ Chile, Santiago, Chile.

\section{Correspondencia} \\ K. B. Rocha \\ Pontificia Universidade \\ Católica do Rio Grande \\ do Sul. \\ Av. Ipiranga 6681, Porto \\ Alegre, $R S$ \\ 90619-900, Brasil. \\ katiabonesrocha@gmail.com
}

\begin{abstract}
The objective of this study was to analyze the association between social class and psychosocial occupational risk factors and self-rated health and mental health in a Chilean population. A cross-sectional study analyzed data from the First National Survey on Employment, Work, Quality of Life, and Male and Female Workers in Chile $(N=9,503)$. The dependent variables were self-rated health status and mental health. The independent variables were social class (neoMarxist), psychosocial occupational risk factors, and material deprivation. Descriptive and logistic regression analyses were performed. There were inequalities in the distribution of psychosocial occupational risk factors by social class and sex. Furthermore, social class and psychosocial occupational risk factors were associated with unequal distribution of self-rated health and mental health among the working population in Chile. Occupational health interventions should consider workers' exposure to socioeconomic and psychosocial risk factors.
\end{abstract}

Social Class; Work; Mental Health

\section{Resumen}

El objetivo fue examinar la relación entre clase social, riesgo psicosocial laboral y la salud autopercibida y mental en Chile. Se trata de un estudio transversal con los datos de la Primera Encuesta Nacional de Condiciones de Empleo, Trabajo, Calidad de Vida y Salud de los Trabajadores y Trabajadoras en Chile ( $N=9.503)$. Las variables dependientes son: salud mental y salud autopercibida. Las variables explicativas son: clase social neo-marxista, factores de riesgo psicosocial y privación material. Se realizaron análisis descriptivos y de regresión logística. Existen desigualdades en la distribución de los factores exposición laboral a riesgos psicosociales, según clase social y sexo. Además, la clase social y los factores de riesgo psicosocial en el trabajo están asociados a una distribución desigual de la salud autopercibida y salud mental entre la población trabajadora en Chile. Las intervenciones en el área de la salud de los trabajadores deben considerar la clase social y los factores de riesgo psicosocial a que están expuestos los trabajadores.

Clase Social; Trabajo; Salud Mental 


\section{Introducción}

Las sociedades industriales han sufrido un cambio en las últimas décadas, marcado principalmente por una mayor flexibilidad en los procesos de producción. En Chile se ha registrado en los últimos 30 años un proceso de transformación socioeconómica, como resultado del paso de un modelo de sustitución de importaciones, liderado por el Estado, a otro abierto, basado en la competencia en el mercado global, y liderado por los sectores privados 1 . Los principales cambios han sido la disminución de los segmentos sociales agrícolas, la disminución de la clase trabajadora manual, la tercerización de la fuerza de trabajo y la burocratización del trabajo asalariado en las organizaciones privadas 2 .

Otro aspecto que hay que considerar con la mayor flexibilidad en el mercado laboral es el aumento del trabajo informal en América Latina. La clase de microempresarios en América Latina tradicionalmente desempeña la función de vincular la economía moderna con la gran masa de trabajadores informales, que están en el tramo inferior del mercado de trabajo, a partir de la producción de bienes y servicios de bajo costo para los consumidores e insumos baratos subcontratados por las empresas grandes ${ }^{3}$. Por otro lado, hay poco conocimiento acerca de cómo este proceso de transformación socioeconómica afectó a la estructura social de clase del país y las oportunidades de vida y salud en los diferentes estratos sociales.

En los últimos años se ha visto un mayor interés de la epidemiología social en investigar la relación de clase social 4,5 y salud. Diferentes estudios muestran que existe una asociación entre clase social y salud 6,7,8,9. En este sentido, la medida de clase social neo-marxista se presenta como una posibilidad de análisis complementario a las medidas clásicas de estratificación social como, por ejemplo, ingresos, ocupacion y educación. La medida de clase social neo-marxista introduce en el análisis las relaciones de propiedad, y relaciones de control y autoridad en el lugar de trabajo, además de las credenciales 10 .

Estudios anteriores muestran que tienen peor salud autopercibida los trabajadores que pertenencen a la pequeña burguesía, los supervisores con menores niveles educativos y los trabajadores manuales, cuando los comparamos con los capitalistas que tienen la propiedad de los medios de producción 10,11. Diferentes estudios también muestran que existe asociación entre clase social y salud mental 10,12,13. En relación a los problemas de salud mental, un estudio de prevalencia en Chile muestra que un poco más de un tercio -(36\%) de la población estudiada- había tenido un desorden psiquiátrico a lo largo de su vida, mientras que uno de cada cinco $(22,6 \%)$ había tenido un desorden en los últimos 6 meses 14. En relación a la salud mental, los indicadores de clase social neo-marxista son especialmente útiles para entender los mecanismos que pueden llevar a los trabajadores a un problema de salud mental. Diferentes estudios muestran que los supervisores básicos, que están subordinados a los gerentes, pero que tienen como atribuiciones contratar, disciplinar, ascender y despedir a otros trabajadores, presentan mayor prevalencia de trastornos mentales comunes que los gerentes y demás trabajadores 11 . En relación con la clase social, es importante tener en cuenta que la asociación entre clase social y salud es diferente entre hombres y mujeres 15,16,17.

En relación con los factores psicosociales de riesgo en el trabajo, estudios muestran que la exposición a factores de riesgo laborales está asociada con peores indicadores de salud 18 . Además, estudios anteriores muestran que existe una asociación entre la clase social de la persona, los factores de riesgo psicosocial a los que está expuesta, y la salud 10,11,19. Estudios muestran que trabajadores no cualificados están expuestos a peores condiciones de trabajo que las personas que pertenecen a otras clases sociales. En este sentido, hay evidencias de que los riesgos físicos y psicosociales del trabajo se concentraron en las posiciones de clase, donde los trabajadores no tienen control sobre los medios de producción, como, por ejemplo, las de los supervisores con nivel educacional bajo y los trabajadores semicualificados y no cualificados 11 .

La Primera Encuesta Nacional de Empleo, Trabajo, Salud y Calidad de Vida de los Trabajadores y Trabajadoras de Chile (ENETS 2009) incluyó temas de clase social y riesgos psicosociales asociados al trabajo, lo que permite analizar la asociación entre estas variables y cómo esta asociación afecta la salud de la población. Es importante destacar que existen pocos estudios que analicen la asociación entre clase social y salud en América Latina 17,20 y no hay estudios que analicen la asociación entre salud, clase social y factores de riesgo psicosociales de manera conjunta en América Latina. Así, el objetivo del presente estudio será analizar la distribución de los factores exposición laboral a riesgos psicosociales, en población trabajadora chilena, según clase social. Además, se analiza cómo la clase social y los factores de riesgo psicosociales en el trabajo pueden estar asociados con la salud autopercibida y mental en la población trabajadora chilena. 


\section{Método}

Diseño, fuente de información y población de estudio

Se trata de un estudio de diseño transversal, utilizando los datos de la ENETS 2009. El diseño de la muestra es probabilístico, estratificado geográficamente, y representativo de la población trabajadora a nivel nacional, y por zonas urbanas y rurales 21 .

La población incluida en la ENETS son los mayores de 15 años que refieren estar ocupados (en la fecha del empadronamiento), más aquellos que en la fecha del empadronamiento estuvieron trabajado los últimos 12 meses, incluyendo los residentes de áreas urbanas y rurales $(\mathrm{N}=9.503)$. Las entrevistas fueron realizadas en el hogar del entrevistado por profesionales entrenados.

Los participantes del presente estudio firmaban un consentimiento informado que explicaba los objetivos del estudio y el uso que se daría a éste. En el consentimiento también se divulgaba una página web del Ministerio de Salud de Chile en la que los participantes podrían tener más información sobre el estudio, formular reclamaciones y obtener información sobre los encuestadores. El organismo que recopiló los consentimientos informados fue el centro de microdatos de la Universidad de Chile. Los datos del presente estudio forman parte de las estadísticas oficiales del Ministerio de Salud de Chile.

\section{Variables de estudio}

\section{- Variables dependientes}

Estado de salud autopercibido, a partir de la pregunta: "En general, ¿ cómo diría usted que está su salud?" fue creada una variable dicotómica. La escala Likert de 5 puntos fue transformada en buena salud autopercibida (muy buena y buena) y mala salud autopercibida (regular, mala y muy mala). Este ítem es una medida adecuada de salud global y es un buen predictor de morbilidad y mortalidad 22. Salud mental: el General Health Questionnaire (GHQ-12) es un instrumento de cribado de salud mental que identifica posibles casos de trastorno mental en la población. El GHQ-12 evalúa el estado de salud mental subjetivo de las personas e identifica estrés psicológico actual (psychological distress) y es más sensible a trastornos de depresión y ansiedad. Las personas que puntuaron 3 ó más puntos en la puntuación GHQ-12 son positivas en el cribado, lo que significa que tienen riesgo de presentar trastornos de salud mental 23 .

\section{- Variables independientes}

Clase social neo-marxista según Eric O. Wright 4: el concepto de clase social neo-marxista se basa en tres dimensiones: relaciones de propiedad, control, y credenciales/habilidades. La dimensión de propiedad se obtiene a partir de dos cuestiones: (1) patrón o empleador (dueño o socio), trabajador por cuenta propia, trabajador dependiente o asalariado, servicio doméstico puertas adentro, servicio doméstico puertas afuera, o familiar no remunerado; y (2) número de personas que trabajan para él o ella. Los trabajadores autónomos ocupan la posición de pequeña burguesía, y quienes trabajan por cuenta propia, que tienen entre 2 y 10 trabajadores, están clasificados como pequeños empresarios y los que tienen más de 10 trabajadores ocupan la posición de empresarios.

Las preguntas utilizadas para definir la dimensión de control y autoridad en el lugar de trabajo fueron 3. Las personas que contestaron que supervisan el trabajo de otros empleados o dicen a otros trabajadores qué trabajo tienen que hacer fueron consideradas supervisoras. Fueron considerados gerentes las personas que contestaron que tenían autoridad para contratar o despedir trabajadores, para cambiar los bienes producidos o los servicios que presta su empresa u organización laboral, y sobre el presupuesto.

El proletariado fue dividido en proletariado con contrato formal e informal, lo que se justifica por la grande cantidad de trabajadores informales en América Latina 3. Este es un punto novedoso del presente estudio, ya que es la primera vez que se incluye esa división en la clasificación de clase social neo-marxista. La dimensión de las credenciales (es decir, los expertos, los trabajadores semicualificados y los trabajadores "no cualificados”) se obtuvo mediante la ocupación del encuestado y su nivel educativo.

La clase social fue operacionalizada en 7 categorías: (1) empresarios (empresarios + medianos empresarios); (2) pequeña burguesía; (3) gerentes (todos independientemente de las credenciales); (4) supervisores (todos independientemente de las credenciales); (5) trabajadores expertos; (6) proletariado formal y (7) proletariado informal.

\section{Factores psicosociales en el trabajo}

La escala de factores de riesgo psicosociales, asociados al trabajo, está compuesta por 8 dimensiones:

a) Demanda psicológica (2 ítems): ¿Con qué frecuencia debe resolver problemas difíciles en su trabajo? Su trabajo exige, igran esfuerzo de concentración y/o atención? 
b) Demanda sensorial y física (21 ítems): relacionados con la exposición a riesgos físicos como por ejemplo: vibraciones; ruido; temperaturas altas y bajas; falta de luz natural y radiaciones; exposición a riesgos químicos y biológicos (humo, polvos, solventes); exposición a riesgos ergonómicos: posturas incómodas, mover objetos pesados, movimientos repetitivos, sobresforzar la voz, vista fija y concentrada, trabajar sentado, trabajar de pie.

c) Control sobre el trabajo, autonomía o libertad en la toma de decisiones (5 ítems): puede usted influir: (1) en la velocidad o rapidez con que debe trabajar; (2) en la cantidad de trabajo. Puede elegir o cambiar: (3) el orden de sus tareas; (4) el método o forma en que realiza su trabajo; (5) ¿puede decidir cuándo tomar un descanso?

d) Apoyo social (2 ítems): ¿ con qué frecuencia (1) sus colegas o compañeros o (2) jefe están dispuestos a escuchar sus problemas relacionados con el trabajo?

e) Doble presencia, en casa y el trabajo (4 ítems): ¿cuán a menudo se enfrenta a las siguientes situaciónes: (1) cuando no está en su casa, ¿̇las tareas domésticas se quedan sin hacer?; (2) cuando está en el trabajo, ¿ipiensa en las tareas domésticas y familiares?; (3) ¿̨hay situaciones en que necesitaría estar en el trabajo y en la casa al mismo tiempo?; (4) ¿Cuán a menudo se enfrenta a la siguiente situación: si hay un problema en su casa, usted deja el trabajo para ir a solucionarlo?

f) Calidad de liderazgo (4 ítems): (1) ¿con qué frecuencia encuentra usted disposición en su jefe directo a escuchar sus problemas relacionados con el trabajo?; ¿en qué medida su jefe directo (2) se asegura de que los trabajadores tengan buenas oportunidades de desarrollo laboral?; (3) ¿es bueno en planificar el trabajo?; (4) ¿es bueno en la solución de conflictos?

g) Desarrollo de habilidades (2 ítems): (1) ¿tiene usted la posibilidad de aprender cosas nuevas a través de su trabajo?; (2) ¿le da su trabajo la oportunidad de desarrollar sus capacidades?

h) Discriminación, acoso y malos tratos (20 ítems): ¿alguien que conozca o usted ha sido (1) objeto de acoso sexual por parte de compañeros de trabajo o superiores?; (2) ¿objeto de acoso sexual por parte de clientes, usuarios u otras personas de su trabajo, excepto compañeros o superiores? (3) ¿víctima de agresión física en su lugar de trabajo?; ¿usted ha sentido que sus derechos han sido menos respetados debido a su: (4) edad; (5) nacionalidad; (6) sexo; (7) raza o grupo étnico de origen; (8) minusvalía o discapacidad; (9) orientación sexual; (10) nivel socioeconómico; (11) preferencias políticas; (12) preferencias religiosas; (13) aspecto físico; (14) al lugar donde vive; (15) llevar menos tiempo en el trabajo que sus compañeros?; ¿con qué frecuencia a usted le ha pasado la siguiente situación: (16) no se le habla o se les dificulta a sus compañeros que hablen con usted; (17) le asignan tareas humillantes; (18) no se le asignan tareas; (19) recibe amenazas (escritas, verbales, telefónicas, por gestos); (20) recibe presiones $\mathrm{u}$ hostigamientos para aumentar sus niveles de productividad?

La escala utiliza muchos ítems del Cuestionario Psicosocial de Copenhague (CoPsoQ), que a su vez es un compendio de instrumentos ampliamente usados y validados en la literatura sobre organización del trabajo y salud. Los ítems de la escala son contestados a través de una escala Likert de 4 puntos (nunca, rara vez, casi siempre y siempre). El análisis de las medias en cada dimensión fue transformado en un marcador de puntuación de 0-100 para homogenizar las puntuaciones, donde una mayor puntuación representa una mayor exposición a factores de riesgo psicosociales en el ambiente laboral. Los análisis de la consistencia interna muestran que los valores del alfa de Crombach estaban alrededor del 0,70 en todas las escalas. Otros estudios desarrollados en Chile con el CoPsoQ también han referido adecuadas características psicométricas 24 .

\section{Privación material en casa}

Pregunta acerca de la posesión de los siguientes bienes: lavadora automática, refrigerador, teléfono fijo, conexión a TV a cable/TV satélite/ TV digital, computador o notebook, conexión a Internet, vehículo de uso particular (auto). Para los análisis multivariados se creó una escala sumando las respuestas de las 7 preguntas, cuyo valor oscila entre 0 y 7 . La escala de 0 hasta 7 fue dividida en terciles.

\section{Análisis de datos}

El primer paso fue describir la distribución en la muestra para todas las variables de estudio. En las variables psicosociales y de privación fue descrita la media de la dimensión y también la media por terciles (25, 50 y 75). La decisión de trabajar con estas variables en terciles se justifica por la no normalidad de las distribuciones a partir de la prueba de Shapiro-Wilk.

Después, se describieron las medias de cada una de las dimensiones de los factores psicosociales según la clase social y se analizaron si existían diferencias significativas entre la media de las dimensiones, según la clase social, a partir de la prueba de Kruskal-Wallis no paramétrica, ya que estas variables no siguen una distribución normal. 
En tercer lugar, se ajustaron modelos bivariados con el cálculo de odds ratio (OR) e intervalos de 95\% de confianza (IC95\%) para verificar la asociación entre las variables dependientes (salud autopercibida y mental) y cada una de las variables independientes separadamente. Posteriormente, se ajustaron modelos multivariados de asociación, a partir del cálculo de OR e IC95\% de las variables dependientes (salud y salud mental) y todas las variables explicativas que fueran significativas en el modelo bivariado, ajustados por edad. Todos los análisis se realizaron por separado para hombres y mujeres, ya que la literatura indica que la asociación entre salud y clase social es diferente entre hombres y mujeres 15,16,17. Todos los análisis fueron ponderados por los pesos derivados de la muestra de diseño complejo.

\section{Resultados}

La Tabla 1 presenta la descripción de la muestra. En relación con los factores de riesgo psicosocial, las dimensiones que tienen las medias más elevadas son la demanda psicológica $(m=59,1$ hombres y $\mathrm{m}=52,4$ mujeres) y la dimensión de control y autonomía $(\mathrm{m}=42,4$ hombres y $\mathrm{m}=$ 44,9 mujeres). Los hombres relatan más que las mujeres estar expuestos a demandas físicas y sensoriales $(\mathrm{m}=23,8$ hombres y $\mathrm{m}=15,3 \mathrm{mu}-$ jeres). Por otro lado, las mujeres refieren más riesgos psicosociales relacionados a la doble presencia ( $\mathrm{m}=17,6$ hombres y $\mathrm{m}=42,1$ mujeres), calidad de liderazgo y la posibilidad de desarrollo de habilidades.

En la Tabla 2 se puede observar que hay diferencias significativas entre las medias de las diferentes dimensiones de factores de exposición laboral a riesgos psicosociales, según clase social. Los factores asociados a demandas psicológicas o cognitivas fueron referidos más por los gerentes y supervisores. Las demandas físicas y sensoriales fueron más referidas por los hombres del proletariado informal $(\mathrm{m}=25,6)$ y por las mujeres supervisoras $(m=25)$. La falta de control y autonomía fueron referidos con más frecuencia por el proletariado formal ( $\mathrm{m}=56,3$ hombres $\mathrm{y}$ $\mathrm{m}=55,7$ mujeres). Entre los hombres las medias más altas de riesgo asociado a la falta de apoyo social aparecen entre la pequeña burguesía y el proletariado informal, y entre las mujeres las mayores medias están en el proletariado informal $(\mathrm{m}=47,5)$ y formal $(\mathrm{m}=38,9)$. En la dimensión doble presencia la mayor puntuación está entre los empresarios, la pequeña burguesía (hombres y mujeres) y supervisoras (mujeres). Entre las mujeres presentan más problemas asociados a la dimensión calidad de el liderazgo, las empresarias y el proletariado formal, lo que indica dificuldades de relación con los jefes. El mayor riesgo psicosocial, asociado a la dimensión -desarrollo de habilidades-, se encuentra en el proletariado formal e informal (hombres y mujeres). En la dimensión discriminación, acoso y malos tratos la mayor puntuación está entre los supervisores (hombres y mujeres).

En la Tabla 3 se presentan los resultados de los análisis de asociación entre la variable dependiente salud y las variables explicativas. Entre los hombres, los análisis del modelo multivariado muestran que la variable que sigue siendo significativa es la de los empresarios que son los que menos refieren tener una mala salud autopercibida (OR $=0,68$, IC95\%: 0,48-0,95), si los comparamos con el proletariado formal. Entre las dimensiones de riesgo psicosocial en el trabajo, que están más asociadas a una mayor prevalencia de mala salud autopercibida, son haber puntuado alto en las dimensiones de doble presencia (Q1: OR = 1,27, IC95\%: 1,09-1,48; Q2: OR = 2,10, IC95\%: 1,72-2,56), demanda física y sensorial (Q1: OR = 1,60, IC95\%: 1,33-1,92; Q2: OR = 1,58, IC95\%: 1,32-1,89), apoyo social (Q1: OR = 1,23, IC95\%: 1,00-1,52; Q2: OR = 1,50, IC95\%: 1,24$1,81)$, desarrollo de habilidades (Q2: $\mathrm{OR}=1,35$, IC95\%: 1,10-1,64) y calidad de liderazgo (Q2: OR = 1,35, IC95\%: 1,10-1,64). Además, cuanto mayor es la privación material en casa, mayor es la prevalencia de mala salud autopercibida.

En las mujeres los resultados del análisis del modelo multivariado muestran que las variables de clase social dejan de ser significativas. Entre las dimensiones de riesgo psicosocial en el trabajo, que están más asociadas a una mayor prevalencia de mala salud autopercibida, están el bajo apoyo social en el trabajo (Q1: OR = 1,71, IC95\%: 1,35-2,16; Q2: OR = 1,63, IC95\%: 1,28-2,08), una elevada demanda física y sensorial (Q1: OR = 1,49, IC95\%: 1,26-1,77; Q2: OR = 1,43, IC95\%: $1,15-1,78)$, una elevada percepción de necesidad de doble presencia (casa y trabajo) (Q2: OR = 1,59, IC95\%: 1,30-1,94), dificultades con el jefe directo, expresadas a partir de una alta puntuación en la dimensión de calidad de liderazgo (Q2: OR $=1,59$, IC95\%: 1,28-1,99) y una elevada demanda psicológica en el trabajo $(\mathrm{Q} 2: \mathrm{OR}=1,30$, IC95\%: 1,02-1,68). Además, cuanto mayor es la privación material en casa, mayor es la prevalencia de mala salud autopercibida.

La Tabla 4 presenta los resultados sobre la mala salud mental (ser positivo en el cribado GHQ). Entre los hombres, los análisis del modelo multivariado muestran que sigue siendo significativo: entre los empresarios (OR = 0,52; IC95\%: 0,35$0,77)$, la pequeña burguesía $(\mathrm{OR}=0,67$, IC95\%: 
Descripción de la población estudiada (número y porcentaje), según variables dependientes e independientes. Población activa (16-64 años).

\begin{tabular}{|c|c|c|c|c|c|c|}
\hline & \multicolumn{3}{|c|}{ Hombres } & \multicolumn{3}{|c|}{ Mujeres } \\
\hline & $\mathrm{n}$ & $\%$ & & $\mathrm{n}$ & $\%$ & \\
\hline \multicolumn{7}{|l|}{ Variables dependientes } \\
\hline Mala salud autopercibida & 941 & 21,3 & & 1.055 & 35,6 & \\
\hline Positivo cribado GHQ-12 & 808 & 18,2 & & 836 & 28,2 & \\
\hline \multicolumn{7}{|l|}{ Variables independientes } \\
\hline \multicolumn{7}{|l|}{ Clase social } \\
\hline Empresarios & 381 & 8,6 & & 141 & 4,8 & \\
\hline Pequeña burguesía & 863 & 19,5 & & 529 & 17,9 & \\
\hline Gerentes & 264 & 6,0 & & 165 & 5,6 & \\
\hline Supervisores & 304 & 6,7 & & 119 & 4,0 & \\
\hline Trabajadores expertos & 438 & 9,9 & & 468 & 15,8 & \\
\hline Proletariado formal & 1.980 & 44,7 & & 1.294 & 43,7 & \\
\hline Proletariado informal & 200 & 4,5 & & 245 & 8,2 & \\
\hline Dimensiones psicosociales & $\mathrm{n}$ & Media & DP & $\mathrm{n}$ & Media & DP \\
\hline Demanda psicológica & 5.636 & 59,1 & 25,4 & 3.764 & 52,4 & 28,0 \\
\hline Q1 & 2.669 & 36,0 & 15,9 & 1.912 & 31,2 & 19,1 \\
\hline Q2 & 2.055 & 68,9 & 6,25 & 1.038 & 68,6 & 6,25 \\
\hline Q3 & 1.174 & 94,3 & 6,22 & 523 & 93,6 & 6,25 \\
\hline Demanda física y sensorial & 5.208 & 23,8 & 14,5 & 3.559 & 15,3 & 10,4 \\
\hline Q1 & 1.307 & 8,9 & 3,06 & 1.680 & 7,9 & 3,48 \\
\hline Q2 & 2.002 & 19,6 & 3,58 & 1.149 & 18,3 & 3,51 \\
\hline Q3 & 2.207 & 39,3 & 10,9 & 520 & 35,2 & 7,83 \\
\hline Control y autonomía & 5.495 & 42,4 & 29,5 & 3.654 & 44,9 & 29,3 \\
\hline Q1 & 2.179 & 9,5 & 9,6 & 1.173 & 9,4 & 9,45 \\
\hline Q2 & 2.007 & 46,0 & 9,9 & 1.286 & 45,6 & 9,94 \\
\hline Q3 & 1.524 & 79,1 & 11,0 & 916 & 80,4 & 10,8 \\
\hline Apoyo social & 4.107 & 35,7 & 29,1 & 2.288 & 37,2 & 29,4 \\
\hline Q1 & 1.535 & 3,40 & 5,56 & 788 & 3,68 & 5,7 \\
\hline Q2 & 1.691 & 38,6 & 10,7 & 948 & 37,6 & 10,7 \\
\hline Q3 & 937 & 76,3 & 13,9 & 552 & 77,0 & 13,5 \\
\hline Doble presencia & 5.608 & 17,6 & 19,1 & 3.677 & 42,1 & 29,8 \\
\hline Q1 & 2.554 & 2,49 & 3,8 & 584 & 2,40 & 3,8 \\
\hline Q2 & 2.393 & 24,2 & 6,3 & 935 & 25,9 & 6,5 \\
\hline Q3 & 910 & 54,9 & 16,2 & 1.913 & 66,1 & 17,7 \\
\hline Calidad de liderazgo & 3.988 & 34,3 & 27,6 & 2.551 & 38,1 & 29,2 \\
\hline Q1 & 1.647 & 6,37 & 7,3 & 891 & 5,7 & 7,2 \\
\hline Q2 & 1.174 & 33,4 & 7,3 & 687 & 33,3 & 6,9 \\
\hline Q3 & 1.234 & 66,4 & 15,9 & 809 & 69,3 & 16,0 \\
\hline Desarrollo de habilidades & 5.331 & 35,3 & 31,8 & 2.551 & 38,1 & 35,2 \\
\hline Q1 & 2.217 & 2,77 & 5,19 & 1.152 & 2,77 & 5,19 \\
\hline Q2 & 1.936 & 38,2 & 10,7 & 992 & 40,5 & 10,5 \\
\hline Q3 & 1.296 & 80,5 & 14,8 & 1.049 & 69,3 & 14,4 \\
\hline Discriminación, acoso y malos tratos & 4.883 & 3,29 & 8,4 & 3.209 & 4,28 & 9,3 \\
\hline Q1 & 3.968 & 0,0 & - & 2.107 & 0,0 & - \\
\hline $\mathrm{Q} 2$ & 1.257 & 11,7 & 12,3 & 895 & 12,9 & 12,1 \\
\hline Privación material en casa $(0-7)$ * & 5.591 & 3,16 & 2,09 & 3.700 & 2,94 & 2,0 \\
\hline Q1 & 2.604 & 1,31 & 1,13 & 1.676 & 1,34 & 1,15 \\
\hline Q2 & 2.285 & 4,51 & 0,50 & 1.337 & 4,58 & 0,49 \\
\hline Q3 & 970 & 6,25 & 0,44 & 437 & 6,19 & 0,39 \\
\hline
\end{tabular}

GHQ-12: General Health Questionnaire.

* Privación material en casa con escala de 0-7, a mayor puntuación, mayor privación.

Nota: media en las dimensiones de factores psicosociales de 0-100. Cuanto mayor es la media, más expuesto a factores de riesgo psicosociales. 
Análisis del marcador de puntuación en cada una de las dimensiones de factores de riesgo psicosociales, según clase social (Wright 4).

\begin{tabular}{|c|c|c|c|c|c|c|c|c|}
\hline & Empresarios & $\begin{array}{l}\text { Pequeña } \\
\text { burguesía }\end{array}$ & Gerentes & Supervisores & $\begin{array}{c}\text { Trabajadores } \\
\text { expertos }\end{array}$ & $\begin{array}{l}\text { Proletariado } \\
\text { formal }\end{array}$ & $\begin{array}{c}\text { Proletariado } \\
\text { informal }\end{array}$ & Valor de $p$ \\
\hline & Media & Media & Media & Media & Media & Media & Media & \\
\hline \multicolumn{9}{|c|}{ Demanda psicológica o cognitiva } \\
\hline Hombres & 66,8 & 58,1 & 71,7 & 71,0 & 65,4 & 54,0 & 51,0 & * \\
\hline Mujeres & 48,7 & 53,2 & 66,0 & 69,6 & 65,2 & 47,1 & 39,0 & * \\
\hline \multicolumn{9}{|c|}{ Demanda física y sensorial } \\
\hline Hombres & 22,6 & 24,2 & 21,1 & 25,2 & 20,0 & 24,7 & 25,6 & * \\
\hline Mujeres & 16,0 & 12,9 & 17,6 & 25,0 & 14,7 & 15,5 & 13,9 & * \\
\hline \multicolumn{9}{|c|}{ Control y autonomía } \\
\hline Hombres & 20,5 & 20,2 & 28,7 & 43,9 & 46,1 & 56,3 & 45,0 & * \\
\hline Mujeres & 28,6 & 24,3 & 32,9 & 47,3 & 47,3 & 55,7 & 42,4 & * \\
\hline \multicolumn{9}{|l|}{ Apoyo social } \\
\hline Hombres & 28,8 & 46,9 & 29,4 & 25,9 & 33,0 & 37,8 & 43,1 & * \\
\hline Mujeres & 27,5 & 39,4 & 32,0 & 32,1 & 34,7 & 38,9 & 47,5 & * \\
\hline \multicolumn{9}{|c|}{ Doble presencia } \\
\hline Hombres & 22,3 & 21,0 & 19,5 & 16,0 & 16,4 & 15,3 & 18,9 & * \\
\hline Mujeres & 41,9 & 29,1 & 30,8 & 38,5 & 34,7 & 41,2 & 33,7 & * \\
\hline \multicolumn{9}{|c|}{ Calidad de liderazgo } \\
\hline Hombres & 22,2 & 45,3 & 27,0 & 31,1 & 32,4 & 35,8 & 40,1 & * \\
\hline Mujeres & 41,9 & 29,1 & 30,8 & 38,5 & 34,7 & 41,2 & 33,7 & * \\
\hline \multicolumn{9}{|c|}{ Desarrollo de habilidades } \\
\hline Hombres & 20,4 & 34,2 & 20,8 & 31,5 & 34,1 & 40,2 & 44,7 & * \\
\hline Mujeres & 36,2 & 32,6 & 28,5 & 38,1 & 31,5 & 49,1 & 54,5 & * \\
\hline \multicolumn{9}{|c|}{ Discriminación, acoso y malos tratos } \\
\hline Hombres & 3,27 & 1,82 & 3,31 & 8,80 & 2,66 & 3,15 & 3,42 & * \\
\hline Mujeres & 1,89 & 2,72 & 4,85 & 9,96 & 4,08 & 4,67 & 3,49 & * \\
\hline
\end{tabular}

* Valor de p significativo.

0,48-0,92) y los trabajadores expertos $(\mathrm{OR}=0,78$, IC95\%: 0,62-0,99) son quienes presentan menor riesgo de tener un problema de salud mental, comparados con el proletariado formal. Entre las dimensiones de riesgo psicosocial en el trabajo las dimensiones que están más asociadas a una mayor prevalencia de mala salud autopercibida son sufrir discriminación, acoso y malos tratos en el trabajo (Q1: OR = 1,78, IC95\%: 1,44-1,19; Q2: OR $=2,76$, IC95\%: 2,34-3,24), tener una elevada demanda física y sensorial (Q1: OR = 1,56, IC95\%: 1,28-1,91; Q2: OR = 1,95; IC95\%: 1,61-2,36), tener poco apoyo social en trabajo $(\mathrm{Q} 1: \mathrm{OR}=1,38$, IC95\%: 1,08-1,77; Q2: OR = 1,28, IC95\%: 1,01$1,62)$, elevada doble presencia $(\mathrm{Q} 2: \mathrm{OR}=2,37$, IC95\%: 1,93-2,91), falta de oportunidades para el desarrollo de habilidades (Q1: OR = 2,06, IC95\%: 1,66-2,55) y dificuldades con el jefe directo (calidad de liderazgo) (OR = 1,50, IC95\%: 1,20-1,88). El tener un mayor control y autonomía es protec- tor para los problemas de salud mental (Q1: OR = 0,72, IC95\%: 0,58-0,87). Además, cuanto mayor es la privación material en casa, mayor es la prevalencia de mala salud mental.

Entre las mujeres, los análisis del modelo multivariado muestran que la clase social de gerentes es la que presenta significativamente mayor probabilidad de tener problemas de salud mental (OR = 2,34, IC95\%: 1,45-3,75). Entre las dimensiones de riesgo psicosocial en el trabajo, que están más asociadas a una mayor prevalencia de mala salud autopercibida, son haber puntuado alto en las dimensiones de doble presencia (Q1: OR = 1,37, IC95\%: 1,05-1,78; Q2: OR $=2,84$, IC95\%: 2,26-3,58) y alta demanda física y sensorial (Q1: OR = 1,47; IC95\%: 1,23-1,77; Q2: OR = 2,74, IC95\%: 2,20-3,42). La privación material en casa sigue siendo una variable muy asociada al riesgo de tener problemas de salud mental para mujeres. 
Modelos multivariados de asociación entre mala salud autopercibida y clase social, factores de riesgo psicosociales en el trabajo y privación material.

\begin{tabular}{|c|c|c|c|c|c|}
\hline & \multirow{3}{*}{$\%$} & \multicolumn{4}{|c|}{ Hombres } \\
\hline & & \multicolumn{2}{|c|}{ Modelo crudo } & \multicolumn{2}{|c|}{ Modelo multivariado * } \\
\hline & & OR & IC95\% & OR ajustada & IC95\% \\
\hline \multicolumn{6}{|l|}{ Clase social } \\
\hline Empresarios & 15,8 & 0,45 ** & $0,33-0,60$ & 0,68 ** & $0,48-0,95$ \\
\hline Pequeña burguesía & 30,0 & 1,03 & $0,84-1,26$ & 1,14 & $0,89-1,47$ \\
\hline Gerentes & 18,9 & 0,56 ** & $0,36-0,87$ & 1,10 & $0,68-1,79$ \\
\hline Supervisores & 13,9 & 0,38 ** & $0,29-0,50$ & 0,87 & $0,64-1,17$ \\
\hline Trabajadores expertos & 17,2 & $0,50 * *$ & $0,41-0,61$ & 0,89 & $0,71-1,10$ \\
\hline Proletariado formal & 29,5 & 1,00 & & 1,00 & \\
\hline Proletariado informal & 30,8 & 1,06 & $0,77-1,45$ & 1,06 & $0,74-1,47$ \\
\hline \multicolumn{6}{|l|}{ Dimensiones psicosociales } \\
\hline \multicolumn{6}{|l|}{ Demanda psicológica } \\
\hline Q1 (referencia) & 23,8 & 1,00 & & & \\
\hline Q2 & 19,3 & $0,80 * \star$ & $0,69-0,93$ & 0,91 & $0,77-1,07$ \\
\hline Q3 & 18,3 & $0,75 * *$ & $0,62-0,92$ & 0,90 & $0,73-1,10$ \\
\hline \multicolumn{6}{|c|}{ Demanda física y sensorial } \\
\hline Q1 (referencia) & 14,5 & 1,00 & & & \\
\hline Q2 & 23,6 & $1,71 * \star$ & $1,43-2,03$ & $1,60 * \star$ & $1,33-1,92$ \\
\hline Q3 & 22,1 & 1,70 ** & $1,43-2,02$ & 1,58 ** & $1,32-1,89$ \\
\hline \multicolumn{6}{|l|}{ Control y autonomía } \\
\hline Q1 (referencia) & 20,3 & 1,00 & & & \\
\hline Q2 & 20,4 & 0,92 & $0,77-1,10$ & & \\
\hline Q3 & 23,5 & 1,12 & $0,92-1,35$ & & \\
\hline \multicolumn{6}{|l|}{ Apoyo social } \\
\hline Q1 (referencia) & 14,0 & 1,00 & & & \\
\hline $\mathrm{Q} 2$ & 19,3 & 1,50 ** & $1,21-1,86$ & 1,23 ** & $1,00-1,52$ \\
\hline Q3 & 29,9 & 1,32 ** & $1,06-1,63$ & $1,50 * \star$ & $1,24-1,181$ \\
\hline \multicolumn{6}{|l|}{ Doble presencia } \\
\hline Q1 (referencia) & 17,4 & 1,00 & & & \\
\hline Q2 & 22,1 & 1,50 ** & $1,16-1,56$ & 1,27 ** & $1,09-1,48$ \\
\hline Q3 & 32,7 & 1,32 ** & $1,98-2,91$ & $2,10 * *$ & $1,72-2,56$ \\
\hline \multicolumn{6}{|l|}{ Calidad de liderazgo } \\
\hline Q1 (referencia) & 14,8 & 1,00 & & & \\
\hline Q2 & 19,2 & $1,34 * *$ & $0,92-1,44$ & 1,18 & $0,99-1,39$ \\
\hline Q3 & 27,2 & $2,40 * *$ & $0,98-1,48$ & 1,35 ** & $1,10-1,64$ \\
\hline \multicolumn{6}{|l|}{ Desarrollo de habilidades } \\
\hline Q1 (referencia) & 16,3 & 1,00 & & & \\
\hline Q2 & 21,2 & 1,15 & $1,10-1,54$ & 1,18 & $0,99-1,39$ \\
\hline Q3 & 26,8 & 1,20 & $1,21-1,81$ & 1,35 ** & $1,10-1,64$ \\
\hline \multicolumn{6}{|l|}{ Discriminación, acoso y } \\
\hline Q1 (referencia) & 18,9 & 1,00 & & & \\
\hline $\mathrm{Q} 2$ & 22,3 & 1,22 ** & $1,00-1,48$ & 1,19 & $0,97-1,46$ \\
\hline Q3 & 26,7 & 1,43 ** & $1,22-1,67$ & 1,58 ** & $1,36-1,86$ \\
\hline \multicolumn{6}{|c|}{ Privación material en casa } \\
\hline Q1 (referencia) & 14,1 & 1,00 & & & \\
\hline $\mathrm{Q} 2$ & 25,4 & 2,07 ** & $1,79-2,39$ & 1,76 ** & $1,50-2,07$ \\
\hline Q3 & 36,7 & 3,55 ** & $2,95-4,27$ & 2,49 ** & $2,00-3,09$ \\
\hline
\end{tabular}

(continua) 


\begin{tabular}{|c|c|c|c|c|c|}
\hline & \multirow{3}{*}{$\%$} & \multicolumn{4}{|c|}{ Mujeres } \\
\hline & & \multicolumn{2}{|c|}{ Modelo crudo } & \multicolumn{2}{|c|}{ Modelo multivariado * } \\
\hline & & OR & IC95\% & OR ajustada & $\mathrm{IC} 95 \%$ \\
\hline \multicolumn{6}{|l|}{ Clase social } \\
\hline Empresarios & 32,5 & 0,67 ** & $0,45-0,99$ & 0,89 & $0,57-1,40$ \\
\hline Pequeña burguesía & 38,6 & 0,87 & $0,66-1,16$ & 0,99 & $0,69-1,42$ \\
\hline Gerentes & 38,6 & 0,87 & $0,57-1,33$ & 1,38 & $0,86-2,21$ \\
\hline Supervisores & 24,8 & 0,46 ** & $0,33-0,64$ & 0,70 & $0,45-1,03$ \\
\hline Trabajadores expertos & 35,0 & 0,74 ** & $0,58-0,96$ & 1,24 & $0,92-1,66$ \\
\hline Proletariado formal & 41,8 & 1,00 & & 1,00 & \\
\hline Proletariado informal & 36,3 & 0,79 & $0,57-1,10$ & 0,99 & $0,70-1,41$ \\
\hline \multicolumn{6}{|l|}{ Dimensiones psicosociales } \\
\hline \multicolumn{6}{|l|}{ Demanda psicológica } \\
\hline Q1 (referencia) & 37,7 & 1,00 & & & \\
\hline Q2 & 31,3 & 0,77 ** & $0,66-0,91$ & 0,87 & $0,73-1,04$ \\
\hline Q3 & 35,0 & 1,06 & $0,85-1,32$ & 1,30 ** & $1,02-1,68$ \\
\hline \multicolumn{6}{|c|}{ Demanda física y sensorial } \\
\hline Q1 (referencia) & 30,7 & 1,00 & & & \\
\hline Q2 & 33,9 & 1,49 ** & $1,26-1,76$ & $1,49 * \star$ & $1,26-1,77$ \\
\hline Q3 & 45,7 & $1,43 * *$ & $1,16-1,77$ & 1,43 ** & $1,15-1,78$ \\
\hline \multicolumn{6}{|l|}{ Control y autonomía } \\
\hline Q1 (referencia) & 35,0 & 1,00 & & & \\
\hline Q2 & 33,5 & 0,94 & $0,78-1,13$ & & \\
\hline Q3 & 38,3 & 1,00 & $0,83-1,23$ & & \\
\hline \multicolumn{6}{|l|}{ Apoyo social } \\
\hline Q1 (referencia) & 25,9 & 1,00 & & & \\
\hline Q2 & 31,5 & $1,99 * \star$ & $1,59-2,48$ & $1,71 \star \star$ & $1,35-2,16$ \\
\hline Q3 & 44,7 & 1,72 ** & $1,36-2,18$ & $1,63 * \star$ & $1,28-2,08$ \\
\hline \multicolumn{6}{|l|}{ Doble presencia } \\
\hline Q1 (referencia) & 27,8 & 1,00 & & & \\
\hline $\mathrm{Q} 2$ & 27,4 & 0,91 & $0,72-1,13$ & 0,87 & $0,69-1,09$ \\
\hline $\mathrm{Q} 3$ & 41,9 & 1,71 ** & $1,41-2,07$ & $1,59 * \star$ & $1,30-1,94$ \\
\hline \multicolumn{6}{|l|}{ Calidad de liderazgo } \\
\hline Q1 (referencia) & 24,2 & 1,00 & & & \\
\hline Q2 & 28,8 & 1,03 & $0,82-1,28$ & 1,10 & $0,85-1,42$ \\
\hline Q3 & 48,6 & 1,37 ** & $1,11-1,70$ & $1,59 * *$ & $1,28-1,99$ \\
\hline \multicolumn{6}{|c|}{ Desarrollo de habilidades } \\
\hline Q1 (referencia) & 30,5 & 1,00 & & & \\
\hline Q2 & 30,2 & 0,90 & $0,75-1,08$ & 0,87 & $0,71-1,05$ \\
\hline Q3 & 43,8 & 1,28 ** & $1,04-1,57$ & 1,23 & $0,99-1,52$ \\
\hline \multicolumn{6}{|l|}{ Discriminación, acoso y } \\
\hline \multicolumn{6}{|l|}{ malos tratos } \\
\hline Q1 (referencia) & 30,7 & 1,00 & & & \\
\hline Q2 & 36,1 & 1,03 & $0,83-1,27$ & 1,04 & $0,83-1,29$ \\
\hline Q3 & 45,3 & 1,65 ** & $1,39-1,86$ & $1,79 * \star$ & $1,49-2,12$ \\
\hline \multicolumn{6}{|c|}{ Privación material en casa } \\
\hline Q1 (referencia) & 31,4 & 1,00 & & & \\
\hline Q2 & 39,2 & 1,40 ** & $1,22-1,61$ & 1,23 ** & $1,05-1,45$ \\
\hline Q3 & 38,7 & 1,91 ** & $1,50-2,44$ & 1,72 ** & $1,03-2,28$ \\
\hline
\end{tabular}

* Modelo ajustado por clase social, demanda psicológica, privación material y edad;

** Valor de p en comparación de clases sociales $<0,05$. 
Modelos multivariados de asociación entre estar en riesgo de tener un problema de salud mental y clase social, factores de riesgo psicosociales en el trabajo y privación material.

\begin{tabular}{|c|c|c|c|c|c|}
\hline & \multirow{3}{*}{$\%$} & \multicolumn{4}{|c|}{ Hombres } \\
\hline & & \multicolumn{2}{|c|}{ Modelo crudo } & \multicolumn{2}{|c|}{ Modelo multivariado * } \\
\hline & & OR & IC95\% & OR ajustada & IC95\% \\
\hline \multicolumn{6}{|l|}{ Clase social } \\
\hline Empresarios & 13,0 & 0,47 ** & $0,34-0,65$ & 0,52 ** & $0,35-0,77$ \\
\hline Pequeña burguesía & 18,9 & $0,74^{\star \star}$ & $0,59-0,93$ & $0,67^{\star \star}$ & $0,48-0,92$ \\
\hline Gerentes & 16,2 & 0,61 ** & $0,38-0,98$ & 0,70 & $0,41-1,21$ \\
\hline Supervisores & 17,6 & 0,68 ** & $0,52-0,88$ & 1,03 & $0,76-1,39$ \\
\hline Trabajadores expertos & 17,3 & 0,66 ** & $0,54-0,81$ & 0,78 ** & $0,62-0,99$ \\
\hline Proletariado formal & 23,9 & 1,00 & & 1,00 & \\
\hline Proletariado informal & 22,5 & 0,92 & $0,66-1,30$ & 0,82 & $0,57-1,20$ \\
\hline \multicolumn{6}{|l|}{ Dimensiones psicosociales } \\
\hline \multicolumn{6}{|l|}{ Demanda psicológica } \\
\hline Q1 (referencia) & 18,3 & 1,00 & & & \\
\hline $\mathrm{Q} 2$ & 17,4 & 1,04 & $0,88-1,22$ & & \\
\hline Q3 & 19,5 & 1,20 & $0,97-1,47$ & & \\
\hline \multicolumn{6}{|c|}{ Demanda física y sensorial } \\
\hline Q1 (referencia) & 12,0 & 1,00 & & & \\
\hline Q2 & 15,8 & 1,62 ** & $1,32-1,97$ & 1,56 ** & $1,28-1,91$ \\
\hline Q3 & 24,7 & $1,97 * \star$ & $1,64-2,38$ & $1,95 * \star$ & $1,61-2,36$ \\
\hline \multicolumn{6}{|l|}{ Control y autonomía } \\
\hline Q1 (referencia) & 17,1 & 1,00 & & & \\
\hline Q2 & 16,8 & $0,77 * *$ & $0,63-0,93$ & 0,72 ** & $0,58-0,87$ \\
\hline Q3 & 22,4 & 0,97 & $0,79-1,20$ & 0,86 & $0,70-1,07$ \\
\hline \multicolumn{6}{|l|}{ Apoyo social } \\
\hline Q1 (referencia) & 11,8 & 1,00 & & & \\
\hline Q2 & 16,1 & $1,28 * \star$ & $1,00-1,62$ & 1,38 ** & $1,08-1,77$ \\
\hline Q3 & 30,3 & 1,24 & $0,98-1,57$ & 1,28 ** & $1,01-1,62$ \\
\hline \multicolumn{6}{|l|}{ Doble presencia } \\
\hline Q1 (referencia) & 10,7 & 1,00 & & & \\
\hline Q2 & 15,8 & 1,03 & $0,87-1,20$ & 1,02 & $0,86-1,19$ \\
\hline Q3 & 28,8 & 2,41 ** & $1,97-2,95$ & 2,37 & $1,93-2,91$ \\
\hline \multicolumn{6}{|l|}{ Calidad de liderazgo } \\
\hline Q1 (referencia) & 10,7 & 1,00 & & & \\
\hline Q2 & 15,8 & 1,20 & $0,94-1,54$ & 1,23 & $0,99-1,75$ \\
\hline Q3 & 28,8 & $1,49 * *$ & $1,19-1,86$ & $1,50 * \star$ & $1,20-1,88$ \\
\hline \multicolumn{6}{|c|}{ Desarrollo de habilidades } \\
\hline Q1 (referencia) & 13,3 & 1,00 & & & \\
\hline Q2 & 16,5 & $1,25 * \star$ & $1,03-1,51$ & 1,17 & $0,97-1,42$ \\
\hline Q3 & 28,7 & 2,27 & $1,83-2,81$ & 2,06 ** & $1,66-2,55$ \\
\hline \multicolumn{6}{|l|}{$\begin{array}{l}\text { Discriminación, acoso y } \\
\text { malos tratos }\end{array}$} \\
\hline Q1 (referencia) & 12,7 & 1,00 & & & \\
\hline $\mathrm{Q} 2$ & 30,9 & 1,78 ** & $1,44-2,19$ & 1,78 ** & $1,44-2,19$ \\
\hline Q3 & 20,6 & 2,66 ** & $1,27-3,13$ & 2,76 ** & $2,34-3,24$ \\
\hline \multicolumn{6}{|c|}{ Privación material en casa } \\
\hline Q1 (referencia) & 15,3 & 1,00 & & & \\
\hline Q2 & 19,9 & 1,35 ** & $1,17-1,57$ & 1,21 ** & $1,02-1,43$ \\
\hline Q3 & 25,0 & 1,84 ** & $1,51-2,24$ & 1,25 & $0,99-1,58$ \\
\hline
\end{tabular}

(continua) 


\begin{tabular}{|c|c|c|c|c|c|}
\hline & \multirow{3}{*}{$\%$} & \multicolumn{4}{|c|}{ Mujeres } \\
\hline & & \multicolumn{2}{|c|}{ Modelo crudo } & \multicolumn{2}{|c|}{ Modelo multivariado * } \\
\hline & & OR & $\mathrm{IC} 95 \%$ & OR ajustada & $\mathrm{IC95 \%}$ \\
\hline \multicolumn{6}{|l|}{ Clase social } \\
\hline Empresarios & 25,5 & $0,67 * *$ & $0,45-0,99$ & 0,84 & $0,52-1,37$ \\
\hline Pequeña burguesía & 31,5 & 0,87 & $0,66-1,16$ & 1,23 & $0,85-1,78$ \\
\hline Gerentes & 43,4 & 0,87 & $0,57-1,33$ & 2,34 ** & $1,45-3,75$ \\
\hline Supervisores & 29,5 & 0,46 ** & $0,33-0,64$ & 1,32 & $0,89-1,94$ \\
\hline Trabajadores expertos & 24,2 & $0,74 * \star$ & $0,58-0,96$ & 1,09 & $0,80-1,48$ \\
\hline Proletariado formal & 34,1 & 1,00 & & 1,00 & \\
\hline Proletariado informal & 33,6 & 0,79 & $0,57-1,10$ & 1,26 & $0,86-1,84$ \\
\hline \multicolumn{6}{|l|}{ Dimensiones psicosociales } \\
\hline \multicolumn{6}{|l|}{ Demanda psicológica } \\
\hline Q1 (referencia) & 26,6 & 1,00 & & & \\
\hline Q2 & 29,3 & 1,06 & $0,89-1,27$ & & \\
\hline $\mathrm{Q} 3$ & 30,5 & 1,24 & $0,98-1,57$ & & \\
\hline \multicolumn{6}{|c|}{ Demanda física y sensorial } \\
\hline Q1 (referencia) & 22,4 & 1,00 & & & \\
\hline Q2 & 29,1 & 1,41 ** & $1,22-1,74$ & 1,47 ** & $1,23-1,77$ \\
\hline Q3 & 50,3 & $2,63 * \star$ & $2,15-3,25$ & $2,74 * \star$ & $2,20-3,42$ \\
\hline \multicolumn{6}{|l|}{ Control y autonomía } \\
\hline Q1 (referencia) & 29,1 & 1,00 & & & \\
\hline $\mathrm{Q} 2$ & 30,1 & 1,10 & $0,90-1,33$ & 1,11 & $0,91-1,36$ \\
\hline Q3 & 24,3 & $0,80 * *$ & $0,64-0,99$ & 0,84 & $0,68-1,05$ \\
\hline \multicolumn{6}{|l|}{ Apoyo social } \\
\hline Q1 (referencia) & 22,1 & 1,00 & & & \\
\hline $\mathrm{Q} 2$ & 28,4 & 1,25 & $0,99-1,58$ & & \\
\hline Q3 & 32,8 & 1,05 & $0,82-1,35$ & & \\
\hline \multicolumn{6}{|l|}{ Doble presencia } \\
\hline Q1 (referencia) & 14,6 & 1,00 & & & \\
\hline Q2 & 20,9 & $1,44 * *$ & $1,11-1,88$ & $1,37 * *$ & $1,05-1,78$ \\
\hline Q3 & 37,4 & $1,56 * \star$ & $1,23-1,97$ & 2,84 ** & $2,26-3,58$ \\
\hline \multicolumn{6}{|l|}{ Calidad de liderazgo } \\
\hline Q1 (referencia) & 16,2 & 1,00 & & & \\
\hline $\mathrm{Q} 2$ & 30,7 & 1,17 & $0,96-1,43$ & $1,49 * *$ & $1,16-1,91$ \\
\hline Q3 & 33,0 & 1,53 ** & $1,23-1,91$ & 1,70 ** & $1,37-2,11$ \\
\hline \multicolumn{6}{|c|}{ Desarrollo de habilidades } \\
\hline Q1 (referencia) & 23,8 & 1,00 & & & \\
\hline Q2 & 26,2 & 1,17 & $0,96-1,43$ & 1,07 & $0,87-1,30$ \\
\hline Q3 & 33,4 & 1,53 ** & $1,23-1,91$ & 1,36 ** & $1,09-1,69$ \\
\hline \multicolumn{6}{|l|}{$\begin{array}{l}\text { Discriminación, acoso y } \\
\text { malos tratos }\end{array}$} \\
\hline Q1 (referencia) & 22,8 & 1,00 & & & \\
\hline Q2 & 36,6 & $1,48 * *$ & $1,19-1,85$ & 1,54 ** & $1,23-1,93$ \\
\hline Q3 & 32,7 & 1,54 ** & $1,29-1,84$ & 1,59 & $1,32-1,91$ \\
\hline \multicolumn{6}{|c|}{ Privación material en casa } \\
\hline Q1 (referencia) & 23,8 & 1,00 & & & \\
\hline Q2 & 31,7 & 1,46 & $1,26-1,70$ & $1,33 * *$ & $1,12-1,58$ \\
\hline Q3 & 42,3 & 2,35 & $1,83-3,00$ & 2,03 ** & $1,53-2,70$ \\
\hline
\end{tabular}

* Modelo ajustado por clase social, demanda psicológica, privación material y edad;

** Valor de p en comparación de clases sociales $<0,05$. 


\section{Discusión}

Este estudio ha puesto en evidencia que existen desigualdades en la distribución de los factores exposición laboral a riesgos psicosociales, según clase social y sexo. La demanda psicológica o cognitiva es el factor de riesgo psicosocial más referido por las clases de gerentes y supervisores. Por otro lado, la exposición a factores de riesgos de demanda física o sensorial, la falta de control y autonomía y la falta de oportunidades para el desarrollo de habilidades fue referida con más frecuencia por la clase trabajadora y por los supervisores. Estos resultados corroboran los resultados de estudios anteriores que muestran que los riesgos físicos y psicosociales del trabajo se concentraron en las posiciones de clase que no tienen control sobre los activos en el lugar de trabajo (supervisores y trabajadores no expetos) 11,19. A pesar de la distribución desigual de los factores de riesgo psicosocial entre las clases sociales y sexo, es importantante destacar que, tanto para hombres como para mujeres, las dimensiones de riesgo psicosocial más asociadas con una peor salud autopercibida y peor salud mental son una elevada exposición a factores de riesgo físicos y sensoriales en el trabajo, como por ejemplo altas tempertauras, vibraciones, ruidos, etc., y otra corresponde a una elevada demanda de doble presencia -en la casa y el trabajo-. Se suman la baja calidad de liderazgo, lo que indica dificuldades con el jefe directo, el bajo apoyo social percibido por parte de los compañeros de trabajo y el jefe, así como las pocas oportunidades para el desarrollo de habilidades en el trabajo.

Lo anterior, permite afirmar entonces clase social y los factores de riesgo psicosocial en el trabajo están asociados a una distribución desigual de salud general y mental entre la población trabajadora chilena. Se puede observar que a partir del presente estudio se confrma lo descrito en la literatura, que el tener una mayor exposición a factores de riesgo psicosociales en el trabajo está asociado a una peor salud autopercibida y a un mayor riesgo de presentar problemas de salud mental 10,11,25,26,27. Cabe destacar, que a partir de los resultados del presente estudio, se destaca que esta asociación entre salud y exposición a riesgos psicosociales está mediada por la clase social a la que la persona pertenece.

Un mayor riesgo de presentar problemas de salud mental está asociado a las dimensiones de demanda física y sensorial, apoyo social, doble presencia, calidad de liderazgo, desarrollo de habilidades y discriminación, acosos y malos tratos. Estos resultados corroboran lo descrito en la literatura, que los factores laborales de riesgo psicosocial como: el exceso de demanda laboral y una menor autonomía en el trabajo, están asociados con mayor prevalencia de problemas de salud mental 11,13,20,27. Otro resultado que confirma lo descrito en la literatura es que personas con menos apoyo social percibido tienen peor salud autopercibida y mental 28,29 y en este caso considerando, específicamente, el apoyo social recibido en el trabajo.

En el presente estudio los hombres refieren tener más demanda física y sensorial que las mujeres, que por otro lado refieren más riesgo asociado a la doble presencia. Sin embargo, tanto en los hombres, como en las mujeres, una mayor puntuación en la dimensión de demanda física y sensorial está asociada con una peor salud autopercibida y un mayor riesgo de problemas de salud mental.

En relación a la doble presencia, diferentes estudios muestran que el problema de la doble presencia es más relevante entre las mujeres, ya que socialmente se considera aún que las tareas domésticas y de cuidado de personas (niños, discapacitados y personas mayores) son más responsabilidad de las mujeres que de los hombres 15,16,30. Los resultados del presente estudio muestran que las mujeres presentan una puntuación mucho más elevada que los hombres, sin embargo, una mayor puntuación en la dimensión de doble presencia está asociada, tanto en mujeres como en hombres, a una peor salud autopercibida y salud mental. Cabe destacar que estos resultados pueden estar relacionados con la entrada de las mujeres en el mercado de trabajo, que tal vez provoque que los hombres tengan que participar más activamente de las tareas domésticas y familiares. Por otro lado, se observa una vida laboral cada vez más intensa y demandante, tanto para hombres como para mujeres, lo que dificulta la conciliación entre la vida personal y laboral.

En la dimensión calidad de liderazgo y desarrollo de habilidades, los hombres que han referido más riesgo psicosocial se concentran entre las posiciones de clase que no tienen control sobre los activos en el lugar de trabajo 10. Por otro lado, las mujeres que han referido un mayor riesgo en este ámbito son las que tienen una mayor autoridad dentro de las organizaciones, como las empresarias y supervisoras. Probablemente, en función de la estructura y de la historia de la sociedad chilena, que aún puede tener muy presente las cuestiones de desigualdad de género, el hecho de que una mujer tenga más poder puede estar asociado a una mayor exposición al estrés. En este sentido, las mujeres que ocupan posiciones de gerencia son las que tienen significativamente mayor riesgo de presentar problemas de salud mental. Por otra parte, la presencia de ma- 
yores puntuaciones en la dimensión calidad de liderazgo, lo que indica dificultades con los jefes, está asociada a una peor salud autopercibida y salud mental entre hombres y mujeres.

En la dimensión discriminación, acoso y malos tratos en el trabajo, los supervisores son los que han referido significativamente mayor riesgo psicosocial asociado a ellos, que podría estar asociado a la localización "contradictoria de clase", que se refiere a la localización en o entre ambas clases, capitalista o empresario y trabajador. Esto incluye principalmente a los supervisores que se comportan como capitalistas, cuando tienen una posición de dominación sobre los otros trabajadores, y al mismo tiempo como trabajadores, cuando están controlados y explotados por los capitalistas en las relaciones productivas 4 . En este sentido, se agrega a esta situación otro dato importante a destacar: las elevadas puntuaciones en la dimensión de discriminación están asociadas a un mayor riego de problemas de salud mental entre hombres y mujeres.

Los resultados muestran que la clase social y los factores de riesgo psicosociales en el trabajo afectan la salud de los trabajadores, evidenciándose las desigualdades en los resultados de salud autopercebida, según la clase social, siendo los hombres empresarios los que presentan los mejores resultados en ambos indicadores. Lo observado refuerza la idea de interdependencia entre las posiciones de clase, en el sentido de que la mejor salud de un grupo puede estar asociada a la peor salud de otros. Los “empleadores" y los “empleados" forman un todo interdependiente, marcado por una relación antagónica. Los trabajadores no tienen acceso a los recursos productivos y deben producir un excedente, la plusvalía que está apropiada por los capitalistas o empresarios en forma de beneficios, que no son distribuidos igualitariamente con la clase trabajadora 4 Esta relación dependiente e interconectada entre empleados y empleadores puede explicar las diferencias en términos de salud en las distintas clases sociales.

En relación con la salud mental, entre los hombres, se observa nuevamente que los empresarios, la pequeña burguesía y los trabajadores expertos son los que refieren significativamente un menor riesgo de presentar problemas de salud mental. La literatura describe que el gradiente de desigualdades por clase social para la prevalencia de trastornos mentales más comunes no siempre es muy claro $31,32,33$, sin embargo, en este estudio se puede confirmar esa relación, considerando que los resultados muestran que hombres de clase social más privilegiada presentan un menor riesgo de tener problemas de salud mental.
Los resultados del presente estudio refuerzan la hipótesis, ya referida en otros estudios 9,34 , de que los riesgos laborales están relacionados con la necesidad del sistema de extraer más de la mano de obra de los trabajadores para generar los beneficios, que por un lado garantizan la salud de las clases más privilegiadas, como los empresarios y gerentes, pero que por otro lado pueden poner en riesgo la salud de la clase trabajadora.

En el relación con la variable de privación material, esta es una variable asociada a la salud autopercibida y al riesgo de presentar problemas de salud mental, tanto por hombres como por mujeres. Las variables estándar de privación material en el hogar están muy asociadas a ingresos y a la clase social a la que pertenecen los individuos 4 .

En el Ministerio de Salud de Chile existen políticas de promoción de salud dirigidas al desarrollo de lugares de trabajo saludables, que incluyen temas de alimentación, actividad física, ambiente libre de humo de tabaco y donde se promueve el bienestar familiar y social de los trabajadores, a través de la protección de riesgos mecánicos, físicos, ambientales y psicológicos ${ }^{35}$. Sin embargo, estas intervenciones sólo centran su atención sobre los individuos y estilos de vida, sin tener una integración real con los determinantes sociales de la salud en los cuales están inmersas las actividades productivas, así como la población (clase social, género, ingresos, lugar de residencia, etnia, etc.), lo cual podría repercutir negativamente en los resultados esperados de esta política 36 .

\section{Limitaciones y fortalezas}

Entre las limitaciones del presente estudio están el tipo de diseño. Por tratarse de un estudio transversal, no es posible establecer la dirección de la asociación entre las variables. Entre las fortalezas del estudio están las características de la muestra que son representativas de todo el país y de las áreas urbanas y rurales de Chile. El diseño de la encuesta que ha permitido incluir diferentes variables que miden clase social, condiciones de empleo y de trabajo, los factores psicosociales y la percepción de salud en Chile. Los resultados del estudio posibilitan una mayor comprensión de la asociación entre clase social, factores de riesgo psicosociales y su asociación con salud y salud mental en población trabajadora chilena, lo que puede estimular que otros estudios similares sean desarrollados en otros países de América Latina. 


\section{Conclusiones}

El presente estudio contribuye para la comprensión integrada de cómo los factores de exposición laboral a riesgos psicosociales se distribuyen en las diferentes clase sociales y afectan de manera diferenciada a salud autopercibida y la salud mental de mujeres y hombres. El análisis de los factores de riesgo psicosocial, según clase social, pueden ser utilizados como herramientas en el momento de plantear intervenciones, e incluso, legislaciones laborales con la intención de disminuir los riesgos psicosociales en el trabajo en las diferentes clases sociales, lo que podría ayudar a disminuir las desigualdades en salud entre clases sociales y entre hombres y mujeres trabajadores.

\section{Resumo}

O objetivo foi analisar a associação entre classe social, fatores psicossociais de risco laboral e saúde autopercebida e saúde mental entre a população trabalhadora chilena. Estudo transversal com os dados da Primera Encuesta Nacional de Condiciones de Empleo, Trabajo, Calidad de Vida y Salud de los trabajadores y trabajadoras en Chile ( $N=9.503)$. As variáveis dependentes são: saúde autopercebida e saúde mental. As variáveis explicativas são a classe social (neo-marxista), os fatores psicossociais de risco laboral e privação material. Foram realizadas análises descritivas e de regressão logística. Existem desigualdades na distribuição dos fatores psicossociais de risco laboral segundo classe social e sexo. Além disso, a classe social e os fatores de risco psicossociais no trabalho estão associados a uma distribuição desigual da saúde autopercebida e saúde mental. As intervenções na área da saúde dos trabalhadores devem considerar a classe social e fatores de risco psicossociais a que os trabalhadores estão expostos.

\section{Colaboradores}

Todos los autores participaron en la concepción y planificación del artículo, análisis e interpretación de los datos, redacción, revisión crítica del contenido intelectual relevante del artículo y aprobación final de la versión para su publicación.

\section{Agradecimientos}

Al Ministerio de Salud de Chile (Minsal) por la ayuda financiera. 


\section{Referencias}

1. Comisión Económica para América Latina y Caribe. Panorama social de América Latina (20002001). Santiago: Comisión Económica para América Latina y Caribe; 2000.

2. Torche F, Wormald G. Chile: entre la adscripción y el logro. In: Franco R, Léon A, Atria R, editores. Estratificación y movilidad en América Latina. Transformaciones estructurales de un cuarto de siglo. Santiago: LOM Ediciones; 2007. p. 339-87.

3. Portes A, Hoffman K. La estructura de clase en America Latina: composición y cambios durante la era neoliberal. Desarrollo Económico 2003; 43:355-87.

4. Wright EO. The Comparative project on class structure and class consciousness: an overview. Acta Sociologica 1989; 32:3-22.

5. Wright EO. Class counts: comparative studies in class analysis. Cambridge: Cambridge University Press; 2000.

6. Muntaner C, Borrell C, Sola J, Marí-Dell'olmo M, Chung H, Rodríguez-Sanz M, et al. Capitalists, managers, professionals and mortality: findings from the Barcelona Social Class and All Cause Mortality Longitudinal Study. Scand J Public Health 2009; 37:826-38.

7. Muntaner C, Borrell C, Vanroelen C, Chung H, Benach J, Jim H, et al. Employment relations, social class and health: a review and analysis of conceptual and measurement alternatives. Soc Sci Med 2010; 71:2130-40.

8. McFadden E, Luben R, Bingham S, Wareham N, Kinmonth AL, Khaw KT. Does the association between self-rated health and mortality vary by social class? Soc Sci Med 2009; 68:275-80.

9. Muntaner C, O'Campo PJ. A critical appraisal of the demand/control model of the psychosocial work environment: epistemological, social, behavioral and class considerations. Soc Sci Med 1993; 36:1509-17.

10. Muntaner C, Borrell C, Benach J, Pasarín MI, Fernandez E. The associations of social class and social stratification with patterns of general and mental health in a Spanish population. Int J Epidemiol 2003; 32:950-8.

11. Borrell C, Muntaner C, Benach J, Artazcoz L. Social class and self-reported health status among men and women: what is the role of work organisation household material standards and household labour? Soc Sci Med 2004; 58:1869-87.

12. Muntaner C, Eaton WW, Diala CC, Kessler RC, Sorlie PD. Social class, assets, organizational control and the prevalence common groups of psychiatric disorders. Soc Sci Med 1998; 47:2043-53.

13. Cortes I, Artazcoz L, Rodriguez-Sanz M, Borrell C. Desigualdades en la salud mental de la población ocupada. Gac Sanit 2004; 18:351-9.

14. Parada BV, Stevenson PR, Saldivia S, Kohn R, Torres S. Estudio chileno de prevalencia de patología psiquiátrica: DSM-III-R/CIDI, ECPP. Rev Méd Chile 2002; 130:527-36.
15. Artazcoz L, Borrell C, Benach J. Gender inequalities in health among workers: The relation with family demands. J Epidemiol Community Health 2001; 55:639-47.

16. Artazcoz L, Borrell C, Benach J, Cortes I, Rohlfs I. Women, family demands and health: the importance of employment status and socio-economic position. Soc Sci Med 2004; 59:263-74.

17. Rocha KB, Muntaner C, González-Rodríguez MJ, Baksai PB, Vallebuona C, Borrell C, et al. Clase social, desigualdades en salud y conductas relacionadas con la salud de la población trabajadora en Chile. Rev Panam Salud Pública 2013; 33:340-8.

18. Moncada S, Utzet M, Molinero E, Llorens C, Moreno N, Galtés A, et al. The copenhagen psychosocial questionnaire II (COPSOQ II) in Spain-A tool for psychosocial risk assessment at the workplace. Am J Ind Med 2014; 57:97-107.

19. Schrijvers CT, van de Mheen HD, Stronks K, Mackenbach JP. Socioeconomic inequalities in health in the working population: the contribution of working conditions. Int J Epidemiol 1998; 27:1011-8.

20. Muntaner C, Rocha KB, Borrell C, Vallebuona C, Ibanez C, Benach J, et al. Clase social y salud en América Latina. Rev Panam Salud Pública 2012; 31:166-75.

21. Instituto de Seguridad Laboral, Dirección del Trabajo, Ministerio de Salud de Chile. Primera Encuesta Nacional de Empleo, Trabajo y Salud: ENETS 2009. Diseño conceptual y metodológico. Santiago: Ministerio de Salud de Chile; 2009.

22. Idler EL, Beymanini Y. Self-rated health and mortality: a review of twenty-seven community studies. J Health Soc Behav 1997; 38:21-37.

23. Rocha KB, Pérez K, Rodríguez-Sanz M, Borrell $\mathrm{C}$, Obiols JE. Propiedades psicométricas y valores normativos del General Health Questionnaire (GHQ-12) en población general española. Int J Clin Health Psychol 2011; 11:125-39.

24. Alvarado R, Marchetti N, Villalón M, Hirmas M, Pastorino MS. Adaptación y análisis psicométrico de un cuestionario para evaluar riesgos psicosociales en el trabajo en Chile: versión media de CoPsoQ. Rev Chil Salud Pública 2009; 13:7-16.

25. Karasek RA, Theorell T, Schwartz JE, Schnall PL, Pieper CF, Michela JL. Job characteristics in relation to the prevalence of myocardial infarction in the US Health Examination Survey (HES) and the Health and Nutrition Examination Survey (HANES). Am J Public Health 1988; 78:910-8.

26. Schnall PL, Pieper C, Schwartz JE, Karasek RA, Schlussel Y, Devereux RB, et al. The relationship between "job strain”, workplace diastolic blood pressure, and left ventricular mass index esults of a case-control study. JAMA 1990; 263:1929-35.

27. Mausner-Dorsch H. Eaton WW. Psychosocial work environment and depression: epidemiologic assessment of the demand-control model. Am J Public Health 2000; 90:1765-70. 
28. Lahuerta C, Borrell C, Rodríguez-Sanz M, Pérez K, Nebot M. La influencia de la red social en la salud mental de la población anciana. Gac Sanit 2004; 18:83-91.

29. Matud P, Carballeira M, López M, Marrero R, Ibáñez I. Apoyo social y salud: un análisis de género. Salud Ment 2002; 25:32-7.

30. Campos-Serna J, Ronda-Pérez E, Artazcoz L, Benavides FG. Desigualdades de género en salud laboral en España. Gac Sanit 2012; 26:343-51.

31. Fryers T, Melzer D, Jenkins R. Social inequalities and the common mental disorders: a systematic review of the evidence. Soc Psychiatry Psychiatr Epidemiol 2003; 38:229-37.

32. Laaksonen E, Martikainen P, Lahelma E, Lallukka T, Rahkonen O, Head J, et al. Socioeconomic circumstances and common mental disorders among Finnish and British public sector employees: evidence from the Helsinki Health Study and the Whitehall II Study. Int J Epidemiol 2007; 36:776-86.
33. Rocha KB, Pérez K, Rodríguez-Sanz M, Borrell C, Obiols E. Prevalencia de problemas de salud mental y su asociación con variables socioeconómicas, de trabajo y salud: resultados de la Encuesta Nacional de Salud de España. Psicothema (Oviedo) 2010; 22:389-95.

34. Lynch JW, Kaplan G. Socioeconomic position. In: Berkman LF, Kawachi I, editors. Social epidemiology. NewYork: Oxford University Press; 2000. p. 13-35.

35. Salinas CJ, Vio Del Rio F. Promoción de la salud en Chile. Rev Chil Nutr 2002; 29:164-73.

36. Solar O, Irwin A. A conceptual framework for action on the social determinants of health. Geneva: World Health Organization; 2007. (Discussion Paper Series on Social Determinants of Health, 2).

Recibido el 09/Oct/2013

Versión final presentada el 25/Feb/2014

Aprobado el 18/Mar/2014 